\title{
A 3-11 GEV RECIRCULATING LINAC FOR MUON ACCELERATION: DESIGN AND SIMULATION*
}

\author{
C. Johnstone ${ }^{\dagger}$, D. Neuffer FNAL, Batavia, IL 60510, USA \\ E. Forest, KEK, Tsukuba, Japan
}

\begin{abstract}
Muon acceleration is one of the most difficult stages to develop for a Neutrino Factory.or a Muon Collider. The large transverse and longitudinal admittances which must be designed into the system, and the rapidity with which acceleration must take place because of muon decay preclude the use of conventional synchrotron design. The current baseline approach employs recirculating linacs (RLAs) with separate, fixed-field arcs for each acceleration turn. Here a 3-11 GeV RLA will be described along with sophisticated optical codes and tools developed specifically to accurately track, physically describe, and assist in designing this complex system.
\end{abstract}

\section{INTRODUCTION}

One of the more difficult stages to develop for an application requiring an intense muon source is acceleration, especially for a Neutrino Factory due to the ultra-large beam sizes and momentum spreads which must be accelerated[1,2]. Because of potentially heavy losses from muon decay, acceleration must occur rapidly[3], but linear acceleration becomes prohibitively expensive above about a GeV. The current baseline approach relies on recirculating linacs with separate, fixed-field arcs for each acceleration turn although work is ongoing and promising for a fixed-field, alternating-gradient (FFAG) accelerator as an alternative[4].

A baseline acceleration scenario has been developed for references [1] and [5] consisting of a 3-GeV linac (Figure 1), which then injects into a 4-turn, 3-11 GeV RLA (using $200 \mathrm{MHz}$ SC RF), followed by a 5-turn, 11-50 GeV RLA (using $400 \mathrm{MHz} \mathrm{RF}$ ). This paper presents a design for a 3-11 GeV RLA and recent work on a CAD-like optical code which accurately simulates this complex system, providing simultaneously a physical layout and raytracing of trajectories in the same coordinate system. Lattice optical parameters are also computed.

\section{DESIGN CRITERIA AND PARAMETERS}

In an RLA, the beam is injected into a linac, accelerated, and returned by arc transports, thereby achieving multiple passes of acceleration through the same linac. Single-pass acceleration must be used until the beam is sufficiently relativistic and the longitudinal beam size $(\delta z-\delta E / E)$ corresponds to a feasible RLA design in terms of machine acceptance and achieving a clean

\footnotetext{
*Work supported by the Universities Research Association, Inc., under contract DE-AC02-76CH00300 with the U.S. Department of Energy. ${ }^{\dagger} \mathrm{cjj} @$ fnal.gov
}

"passive" separation of the beam on each pass. (Since the circulation time is on the order of a microsecond, an active kicker system is not considered feasible for sorting the beam into different return arcs for transport.) At the end of each arc, the separate transport trajectories are recombined for acceleration through the next linac. The difficulty in designing the lowest-energy recirculating linac lies in directing a beam with both a large transverse emittance and a large momentum spread into separate arcs on each acceleration pass. Beam sizes and momentum spreads used in designing this RLA were taken from reference [1] and are given here in Table 1.

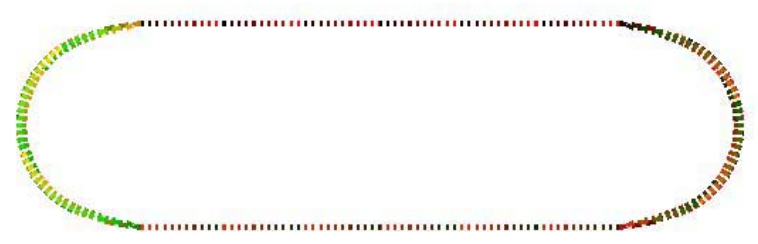

Figure 1. Floor layout of the 4-turn, 3-11 GeV RLA showing the two opposing linacs .

Table 1. Momentum spreads and beams sizes per acceleration pass in a 3-11 GeV RLA.

\begin{tabular}{|c|c|c|c|}
\hline $\begin{array}{c}\text { Central } \\
\text { Momentum } \\
(\mathbf{G E V})\end{array}$ & $\begin{array}{c}\mathbf{\delta p} / \mathbf{p} \\
(\mathbf{3 \sigma}, \mathbf{\%})\end{array}$ & $\begin{array}{c}\text { Momentum } \\
\text { Spread } \\
\mathbf{( G e V )}\end{array}$ & $\begin{array}{c}\text { Beam } \\
\text { Size } \\
(\mathbf{3 \sigma}, \mathbf{c m})\end{array}$ \\
\hline 3 & Injection & & \\
\hline 4 & \pm 5.1 & $3.8-4.2$ & \pm 5.5 \\
\hline 6 & \pm 4.2 & $5.7-6.3$ & \pm 4.8 \\
\hline 8 & \pm 3.8 & $7.7-8.3$ & \pm 4.4 \\
\hline 10 & \pm 3.5 & $9.6-10.4$ & \pm 4.1 \\
\hline 11 & Extraction & & \\
\hline
\end{tabular}

\section{BEAM SPREADER}

A passive beam spreader is used to separate the different energy turns of the RLA into independent magnetic channels: 4, 6, 8, and $10-\mathrm{GeV}$ channels on one side and 5, 7 , and $9 \mathrm{GeV}$ on the opposing side giving a total of 7 return arcs (11-GeV beam may be extracted upstream of the spreader using a kicker and empty linac cells.) The extremely large momentum spreads of Table 1 make a 
clean separation into individual channels difficult, particularly at the highest energies. If the magnets are staggered between neighboring channels, more than $0.5 \mathrm{~m}$ of separation is required to interleave normal-conducting magnets. Here, superconducting magnets are assumed which share collars/yokes (a multiple bore system) allowing the separation to be decreased to $0.4 \mathrm{~m}$. This magnet configuration requires the arcs to be nested as in Figure 1 and parallel; the magnets must be aligned radially and encased in a single cryostat crossing all channels.

In order to avoid matching which is virtually inconceivable for the range in energy over a cycle, a fixed periodic structure (in this case, FODO) is imposed on the entire lattice. Quadrupole gradients remain fixed, as in a non-scaling FFAG, with focusing strength decreasing linearly from the lowest to the highest momentum channel. In order to split the beam into different channels, an extraction-like system is used. If dipole (kicker) spreaders are properly embedded into the FODO structure, then the natural peak excursion locations in the FODO structure can be utilized to enhance the channel separation process. The quadrupoles in the FODO cell are far more effective in beam separation than simple dipoles: the $10 \mathrm{GeV}$ orbit is assumed centered on the spreader quadrupoles to provide a reference orbit and offmomentum orbits are "kicked" relative to the $10 \mathrm{GeV}$ orbit off center through the quadrupoles by the spreader dipoles. The kick increases the farther off-center the trajectory, or the lower the momentum. Channels are separated consecutively from 4 to $8 \mathrm{GeV}$ from the 10 $\mathrm{GeV}$ reference just upstream of every horizontally focusing quadrupole. Septum magnets are inserted prior to a channel separation to "straighten" the rapidly diverging beam to keep the orbits relatively parallel. Once a particular beam energy or channel is separated and begins traversing its own magnetic field channel, another spreader dipole is inserted into the remaining combined channels to facilitate their separation. There are a total of three total spreader dipoles to split off the three lower energy channels from the $10-\mathrm{GeV}$ one. This optimal layout of bends and quadrupoles achieves complete separation of all channels in under $20 \mathrm{~m}$. Figures 2 and 3 show the spreader optics at $4 \mathrm{GeV}$ and $10 \mathrm{GeV}$, respectively.

It is clear that an accurate optical simulation of this complex system with its competing, interleaved elements belonging to different arcs must take into account the physical layout and potential interferences. Prior to this work, optics descriptions and tracking through the separate return transports were performed only in the context of consecutive beamlines with intervening linacs. There was no mechanism to change optics and beam spreader parameters and automatically check for trajectory mismatches to channels, interference between the channels (particularly in the spreader), or even optimize. Recent work[6] has concentrated on developing a CAD-like, highly-accurate (high-order) combined raytracing and lattice code to not only correctly track trajectories through elements relative to a fixed "floor" coordinate system, simultaneously yielding the required optical constants such as beta functions, momentum compaction, etc. The floor layout of Figure 1 and the beam spreader trajectories shown in more detail in Figure 4 for the spreader were produced with this CAD-like optics code. This code has the further strength that elements can be "moved" in a CAD-like fashion, the input optics deck regenerated, and the trajectories automatically retraced. Recognition of conflicts and limiting parameter spaces become immediately available. The accurate degree of "parallelness" of the nested arcs in Figure 1 was achieved by an automated realignment of rough, handcalculated positions for the elements in the individual arcs by this code.

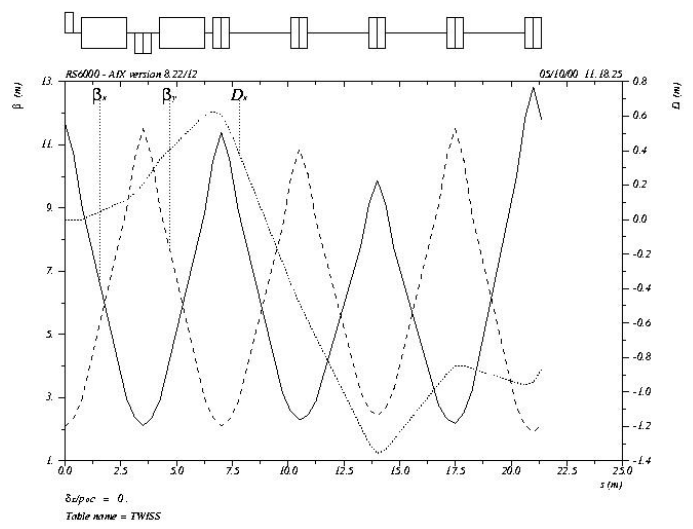

Figure 2. Lattice functions through the beam spreader for 4-GeV beam.

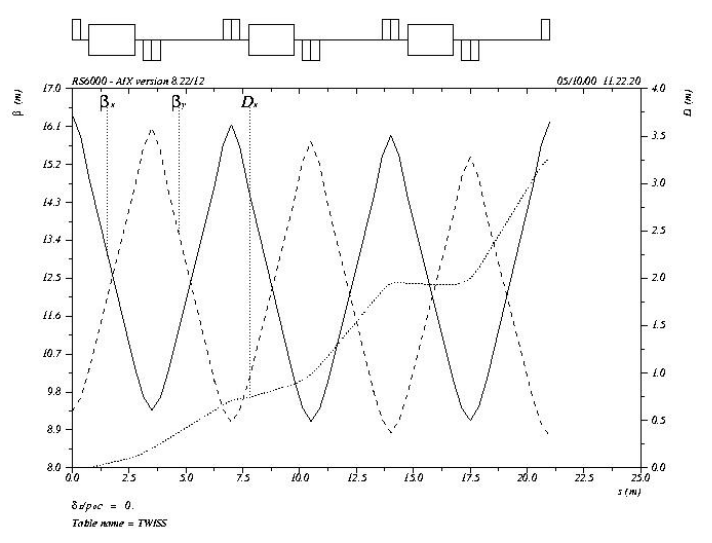

Figure 3. Lattice functions through the beam spreader for $10-\mathrm{GeV}$ beam.

In more detail, the design of the RLA is being checked and simulated with a code that properly separates the local coordinates used on the magnets from the global coordinates of space. This allows us to manipulate magnets and beam lines freely in space. In addition, this code is linked with a polymorphic Taylor series package, 
which permits computation of perturbative quantities. The entire code is written Fortran90, including a revamped version of the differential algebra package in COSY Infinity code[7].

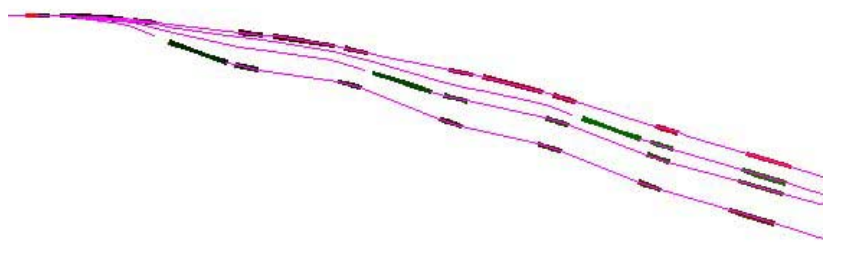

Figure 4. Trajectories traced from the linac through the spreader showing 3 spreader dipoles (purple rectangles), 3 septa magnets (green rectangles) for the consecutive 4, 6, and $8-\mathrm{GeV}$ channel separation. The $4-\mathrm{GeV}$ channel is innermost and $10-\mathrm{GeV}$ outermost. The spreader is approximately $20 \mathrm{~m}$ in length.

\section{THE ARCS}

To design a machine without an active (pulsed section) to match the different arc channels to the linac optics requires that a suitable periodic structure be imposed throughout the machine. Short FODO cells have a large acceptance and small transverse beam sizes. For the linac part, $0.75 \mathrm{~m}$ drifts between magnets for $\mathrm{rf}$ cavities are used as the basic structure (such drifts can be increased to approximately $4 \mathrm{~m}$ without disturbing the optics significantly). This structure is continued through the spreader with the insertion of spreader dipoles or septum magnets in the drift. The arc cells are composed of $1.5 \mathrm{~m}$ gradient dipole magnets with the same cell (focal length), and phase advance as in the linac part (no matching required). Adjustment of the total and relative bend in the arc magnets allows control over the $\mathrm{M}_{56}$ value in each arc as a function of momentum. (The $\mathrm{M}_{56}$ value ranges from 0.5 at $4 \mathrm{GeV}$ to 1.2 at $10 \mathrm{GeV}$ for a $\phi_{\mathrm{s}}$ of $30^{\circ}$.)

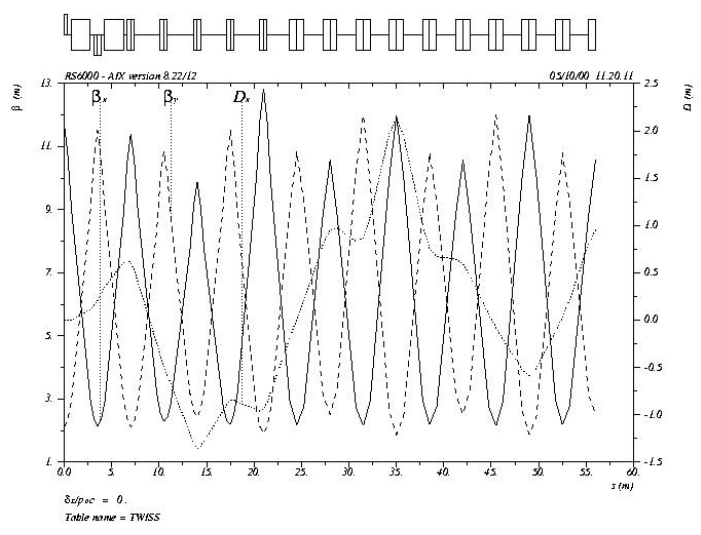

Figure 5. Lattice functions through the beam spreader and first dispersion period for $4-\mathrm{GeV}$ beam.

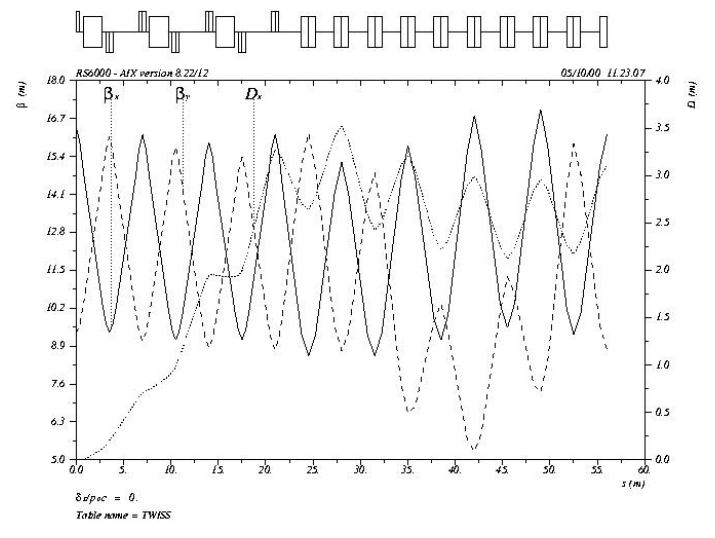

Figure 6. Lattice functions through the beam spreader and first dispersion period for $10-\mathrm{GeV}$ beam.

A linear approach was taken in the control of both dispersion suppression (recombination of trajectories in the linac) and $\mathrm{M}_{56}$. Suppression of linear dispersion is achieved by using an integer phase advance across all arcs. For example, 2490 cells at $4 \mathrm{GeV}$, gives a phase advance of $12,8,6$ and $4.8 \pi$ for $4,6,8$, and $10 \mathrm{GeV}$, respectively. (The $10-\mathrm{GeV}$ arc gradients are slightly adjusted to make the advance $5 \pi$.) The $\mathrm{M}_{56}$ is controlled by adjusting the relative distribution of the dipole strength between the horizontally focusing and vertically focusing gradient magnets in an arc cell, but leaving the total bend of the cell unchanged. Only the derivative of $\mathrm{M}_{56}$ is controlled with a nonlinear element: a single sextupole family in the arcs. $\left(\mathrm{M}_{56}\right.$ ' with respect to momentum is set equal to 0 .)

\section{REFERENCES}

[1] N. Holtkamp and D. Finley, eds., "A Feasibility Study of a Neutrino Source Based on a Muon Storage Ring," Fermilab-Pbu-00/108-E (2000)..

[2] S. Ozaki, R. Palmer, M. Zisman, and J. Gallardo, "Feasibility Study II of a Muon-Based Neutrino Source", BNL-52623, June 2001.

[3 The $\mu^{+} \mu^{-}$Collaboration, $\mu^{+} \mu^{-}$Collider: A Feasibility Study, BNL-52503, Fermi-Conf-96-/092, LBNL-38946, July 1996].

[4] C. Johnstone, "Recent Studies of FFAG Acclerators in the USA," Cyclotrons 2001, East Lansing, MI, May 2001.

[5] D. Neuffer, Fermilab Note, MuCool-75 (2000), and C. Johnstone, "A 3-11 GeV Recirculating Linac for Muon Acceleration," NuFact00, Monterrey, CA, May 2000.

[6] E. Forest, unpublished

[7] M. Berz, COSY INFINITY Version 8 reference manual. http://cosy.nscl.muu.edu. 1996. 